\title{
Atomic scale spin-dependent STM on magnetite using antiferromagnetic STM tips
}

\author{
S. Murphy", S. F. Ceballos, G. Mariotto, N. Berdunov, K. Jordan, I. V. Shvets \\ SFI Nanoscience Laboratories, Physics Department, Trinity College, Dublin 2, \\ Ireland
}

Y. M. Mukovskii

Moscow State Steel and Alloys Institute, Moscow 117936, Russia

\begin{abstract}
STM tips made from antiferromagnetic $\mathrm{MnNi}$ have been used to investigate the atomic structure of the (001) and (111) surfaces of $\mathrm{Fe}_{3} \mathrm{O}_{4}$. The clean (001) surface displays a $(\sqrt{ } 2 \times \sqrt{ } 2) \mathrm{R} 45^{\circ}$ superlattice, which is attributed to charge-ordering on the surface, where $\mathrm{Fe}^{2+}-\mathrm{Fe}^{2+}$ and $\mathrm{Fe}^{3+}-\mathrm{Fe}^{3+}$ dimers can be discriminated. The oxygenterminated (111) surface is characterized by an hexagonal superlattice with a periodicity of $42 \AA$. Oxygen vacancies are observed in atomically resolved images of this superlattice. In the presence of an external magnetic field of $60 \mathrm{mT}$, a significant change in the atomic corrugation in the topmost oxygen layer around each of these defects is observed. The results on both (001) and (111) surfaces are discussed in terms of possible spin polarized effects.
\end{abstract}

PACS: 72.25.-b; 75.70.Rf; 68.37.Ef

Keywords: spin-polarized STM; MnNi; antiferromagnet; magnetite

\section{Introduction}

The advent of scanning tunneling microscopy (STM) in the early 80's can be compared with the impact of low-energy electron diffraction (LEED) on the surface science community in the previous decade. STM has proven to be a powerful tool with a wide range of applications from chemisensitive spectroscopy (STS), to spinpolarized STM and STS of magnetically ordered systems, to STM induced luminescence, to atom-scale manipulation and tip-induced chemistry. Spin-polarized STM (SP-STM) has been one of the more difficult techniques to develop. However, the potential rewards are more than considerable.

The SP-STM technique offers the possibility to achieve magnetic contrast with a resolution on the atomic scale. The technique is derived from the dependence of the tunnel current between two magnetic electrodes, separated by a non-magnetic tunneling barrier, on the alignment of their respective magnetizations (Slonczewski, 1989). In a ferromagnet, the exchange interaction splits the electron states into spin-up and spin-down sub-bands. As a consequence, the local density of states at a given energy will be different for each sub-band. This results in a net polarization $P$ of any current drawn from the ferromagnet near the Fermi level, given by:

\footnotetext{
*Corresponding author. Fax: +353 1608 3228; E-mail address: shmurphy@tcd.ie
} 


$$
P=\frac{J_{\uparrow}-J_{\downarrow}}{J_{\uparrow}+J_{\downarrow}}
$$

where $J \uparrow$ and $J_{\downarrow}$ are the components of the current density from the spin-up and spindown sub-bands respectively. For a ferromagnet-barrier-ferromagnet heterojunction, one can define two polarizations $P_{f b}$ and $P_{b f^{\prime}}$ corresponding to the two ferromagnetbarrier couples present. The resulting conductance $G$ of the junction will take the form:

$$
G=G_{f b f^{\prime}}\left(1+P_{f b} P_{b f^{\prime}} \cos \theta\right)
$$

where $G_{f b f^{\prime}}$ is the mean conductance and $\theta$ is the angle between the magnetization directions of the two electrodes. When the magnetizations of the electrodes are parallel, the tunnel current is maximized, while for the antiparallel configuration, it is minimized. This can be put more simply for the case of tunneling between two ferromagnets, each with $100 \%$ polarization. When the magnetizations are parallel an electron can tunnel between like states on either side of the barrier. However, when the magnetizations are antiparallel, there is no like state for the electron on the opposite side of the barrier and tunneling will not occur.

The initial model was reported for tunneling between two planar ferromagnetic electrodes, but was later adapted to describe the spin-polarized tunneling effect between a ferromagnet and an antiferromagnet in a STM junction (Minakov and Shvets, 1990; Molotkov, 1992). For spin-polarized STM applications, the use of an antiferromagnetic (AF) tip carries distinct advantages over using a ferromagnetic (FM) tip. AF tips do not produce a stray magnetic field, which can affect the sample magnetization of soft ferromagnetic surfaces. Conversely, the tip magnetization cannot be affected by stray fields from the sample, or by an applied magnetic field. Moreover, the tip and sample do not interact through dipolar forces, so that the tunnel gap will be more stable.

As a result, $\mathrm{AF}$ materials like $\mathrm{Cr}$ and $\mathrm{MnNi}$ have been proposed as attractive tip materials for spin-polarized STM applications (Minakov and Shvets, 1990; Shvets et al., 1992). The advantages of using AF tips for non-perturbative imaging were demonstrated by comparing the influence of (FM) GdFe-coated and (AF) Cr-coated $\mathrm{W}$ tips in measurements on the domain structure of Fe bilayer nanowires grown on W(110) (Kubetzka et al., 2002). The stray field from the FM tip is equivalent to an additional 200-300 $\mathrm{mT}$ perpendicular field, which is sufficient to locally trigger remagnetization in the film in external fields that are close to its coercivity. In the present study, we describe atomically resolved SP-STM experiments performed on the magnetite (001) and (111) surfaces, using STM probes made from the antiferromagnetic alloy MnNi.

\section{Materials and Methods}

\section{MnNi STM tips}

$\mathrm{MnNi}$ is one of a family of alloys displaying antiferromagnetism in a narrow composition range $( \pm 2 \%)$ around the equiatomic composition (Kasper and Kouvel, 
1959; Pearson et al., 1965). Other alloys in this family include $\mathrm{MnPt}, \mathrm{MnAu}$ and MnIr. The significant feature of these alloys is their high Néel temperature, which typically lies well above room temperature e.g. $\mathrm{T}_{\mathrm{N}}=800 \pm 40{ }^{\circ} \mathrm{C}$ for $\mathrm{MnNi}$ (Pál et al., 1968), making these materials useful for application in spin-polarized STM in a wide temperature range. $\mathrm{MnNi}$ is a mechanically hard material with a tetragonal $\mathrm{CuAu}-\mathrm{I}$ type structure and the lattice parameters a = 3.74 $\AA$ and $c=3.52 \AA$ (Pál et al., 1968). The crystal can be visualized as a stacking sequence of alternating Mn (001) and $\mathrm{Ni}$ (001) layers. Neutron diffraction experiments have shown that the magnetic moments in each of these planes are antiferromagnetically coupled in-plane. The magnetic moment of the Mn atoms at up to $4.0 \mu_{\mathrm{B}}$ is large, while that of the Ni atoms at $<0.6$ $\mu_{\mathrm{B}}$ is much smaller (Kasper and Kouvel, 1959; Sakuma, 1998).

Polycrystalline ingots of this material were prepared by arc-melting the high purity constituent powders under an argon atmosphere. The ingots were arc-melted 30-40 times to ensure homogeneous mixing of the components, after which they were annealed for $48 \mathrm{~h}$ at $900{ }^{\circ} \mathrm{C}$ under an argon atmosphere. The crystal structure and lattice parameters were verified by powder X-ray diffraction. The ingots were cut into rods using a low-speed diamond wheel saw and mechanically polished into cylindrical shape. The resulting rods were approximately $10 \mathrm{~mm}$ long and $0.6 \mathrm{~mm}$ in diameter.

The MnNi rods were electrochemically etched using the set-up shown schematically in Figure 1. Typically, a dc voltage of 1-4 V was used to drive the cell. The lower portion of the MnNi rod is covered with a close-fitting section of polytetrafluoroethylene (PTFE) tubing (Iijima and Ysuda, 1988; Ceballos et al., 2003). The rod is immersed in a bath of etchant until the meniscus is just above the PTFE. This restricts the active etching region, so that the tip is formed within the upper part of PTFE tube. The dropped portion of the MnNi rod is retrieved for use. It is protected by the PTFE sheath as it drops to the bottom of the bath. Figure 1 also shows scanning electron microscope (SEM) micrographs of an example of the $\mathrm{MnNi}$ tips obtained with this method.

One consideration when producing STM tips from an alloy is the potential impact of the etching procedure on the stoichiometric composition near the tip apex. Auger electron spectroscopy (AES) was used to investigate the impact of the final invacuum ion-etching treatment on the tip stoichiometry. A polished MnNi disk was etched under similar conditions to those used to prepare a STM tip. AES analysis indicated that some preferential sputtering occurs, resulting in a Ni-rich phase (Ceballos et al., 2003). While this suggests that a thin ferromagnetic layer may be produced at the tip apex, it still allows the possibility for spin-polarized tunneling to be obtained.

To investigate the suitability of these tips for SP-STM experiments, several $\mathrm{MnNi}$ tips were tested in SP-STS experiments on $\mathrm{Mn} / \mathrm{Fe}(001)$ epitaxial films. It has been shown that above a coverage of $3 \mathrm{ML}$, the pure ferromagnetic $\mathrm{Mn}(001)$ layers display an in-plane antiferromagnetic interlayer coupling (Tulchinsky et al., 2000; Pierce et al., 2000). Yamada et al. (2003) reported a magnetic contrast of up to $10 \%$ in SP-STS measurements on 7-10 atomic layer thick Mn(001) films, using Fe-coated W tips. Similar experiments carried out under the same conditions, using MnNi tips produced a polarization value of $3 \%$, demonstrating that spin-polarized tunneling can be achieved with these tips. 


\section{Magnetite (001) and (111)}

Magnetite, $\mathrm{Fe}_{3} \mathrm{O}_{4}$, has recently attracted significant attention as a material for spin electronics applications because it is theoretically predicted to be a half-metallic ferromagnet (Zhang and Satpathy, 1991). In simple terms, the exchange interaction is sufficient to split the d-band so that the minority spin sub-band is displaced energetically above the Fermi level $\mathrm{E}_{\mathrm{F}}$, while the majority spin sub-band is lowered below $\mathrm{E}_{\mathrm{F}}$. As a result, any current drawn near the Fermi level should have $100 \%$ spin polarization.

Magnetite is an inverse spinel material based on a face-centered-cubic sublattice of $32 \mathrm{O}^{2-}$ anions, containing $8 \mathrm{Fe}^{3+}$ cations in tetrahedrally coordinated interstices (the so-called A-sites) and a mixture of $8 \mathrm{Fe}^{2+}$ and $8 \mathrm{Fe}^{3+}$ cations in octahedrally coordinated interstices (the so-called B-sites). The crystal structure is illustrated in Figure 2. The lattice parameter of the unit cell is $8.3963 \AA$ (Wyckoff, 1965). Magnetite is a ferrimagnetic material with a Curie temperature of $T_{C}=850 \mathrm{~K}$. The magnetic moments of the cations occupying the A- and B-sites are opposite, where the $\mathrm{Fe}^{3+}$ ions have a magnetic moment of $5 \mu_{\mathrm{B}}$, while the $\mathrm{Fe}^{2+}$ ions have a moment of $4 \mu_{\mathrm{B}}$. This gives a net magnetic moment of $4 \mu_{\mathrm{B}}$ per formula unit, $\mathrm{Fe}^{3+}{ }_{\mathrm{A}}\left[\mathrm{Fe}^{2+} \mathrm{Fe}^{3+}\right]_{\mathrm{B}} \mathrm{O}^{2-}{ }_{4}$. Minority spin electrons hopping between $\mathrm{t}_{2 \mathrm{~g}}$ orbitals on the Bsite cations, are responsible for the high room-temperature electrical conductivity $(\sim$ $\left.200 \Omega^{-1} \mathrm{~cm}^{-1}\right)$. Magnetite undergoes a metal-insulator transition where this electron hopping mechanism is effectively frozen and a charge ordered state is established. The temperature at which this transition occurs is called the Verwey transition temperature $\mathrm{T}_{\mathrm{V}}$, which lies at around $120 \mathrm{~K}$ for bulk stoichiometric magnetite (Verwey et al., 1947; Walz, 2002). In the itinerant electron state above $\mathrm{T}_{\mathrm{V}}$, the B-site cations have a net valence of $\mathrm{Fe}^{2.5+}$, while in the charge ordered state below $\mathrm{T}_{\mathrm{V}}$, a static arrangement of $\mathrm{Fe}^{2+}$ and $\mathrm{Fe}^{3+}$ cations can be discriminated at the B-sites. A number of models have been proposed for this low-temperature $\left(\mathrm{T}<\mathrm{T}_{\mathrm{V}}\right)$ state $($ Kucza, 2001).

Scanning tunneling microscopy studies of the B-terminated (001) surface have suggested that a charge ordered state is established at the surface at a much higher temperature, at least above $300 \mathrm{~K}$ (Coey et al., 1993; Koltun et al., 2001; Mariotto et al., 2002). This localization of charge results in a static arrangement of $\mathrm{Fe}^{2+}$ and $\mathrm{Fe}^{3+}$ cations at the surface. As a result, this surface is particularly suited for atomic scale SP-STM measurements, since the magnetic moment of the $\mathrm{Fe}^{2+}$ and $\mathrm{Fe}^{3+}$ cations differ. The first SP-STM experiments on this surface, carried out by Wiesendanger et al. (1992) using an Fe tip, resolved $<110>$ oriented atomic rows separated by $6 \AA$, with a $12 \AA$ periodicity in corrugation along them. This $12 \AA$ periodicity was not observed when the $<110>$ rows were resolved with a W tip. It was therefore interpreted as a spin-polarized effect, where the ordering of the different magnetic ions was resolved with the Fe tip, but could not be resolved with the paramagnetic W tip. The proposed model of the surface is shown in Figure 3. This interpretation was based on the bulk charge ordering models of Mizoguchi (1978a, b), Iida et al. (1982; 1983) and Kita et al. (1983), which propose static arrangements of B-site cation groups with $12 \AA$ periodicity along the $<110>$ crystallographic directions. Similar results were obtained by Koltun et al. (2001), who also used an Fe tip to image the $\mathrm{Fe}_{3} \mathrm{O}_{4}(001)$ surface. 
These results have stimulated the investigation of the other low-index surfaces of magnetite. Not much information is available in the case of $\mathrm{Fe}_{3} \mathrm{O}_{4}$ (110). Two distinct (110) planes may be identified in the $\mathrm{Fe}_{3} \mathrm{O}_{4}$ bulk, which alternate along the [110] diection with an interlayer separation of $1.484 \AA$. Both layers contain an arrangement of $\mathrm{Fe}$ and $\mathrm{O}$ ions, but while the first contains only B-site cations, the second contains antiferromagnetically coupled A-site and B-site cations. This has prompted interest for spin-polarized STM experiments. However, an STM study by Jansen et al. (1995) has shown that the surface reconstructs into rows aligned along the [-110] crystal direction, and that the resulting surface cannot be reconciled with either of the bulk (110) planes.

In the case of the $\mathrm{Fe}_{3} \mathrm{O}_{4}$ (111) surface, it is possible to identify six (111) layers in the bulk, shown in Figure 4, with a repeat distance of $4.8 \AA$ between equivalent layers. Magnetite has six possible terminations in the [111] direction (Ahdjoudj et al., 1999). Two of these comprise the close-packed oxygen layers $\left(\mathrm{O}_{1}\right.$ and $\left.\mathrm{O}_{2}\right)$, while the others contain $\mathrm{Fe}$ ions. There are major differences between the four Fe layers. The first consists of A-site cations arranged in an hexagonal lattice with a $6 \AA$ interatomic periodicity $\left(\mathrm{Fe}_{\text {tet } 1}\right)$. The second consists of $\mathrm{B}$-site cations arranged in a Kagome lattice with $3 \AA$ interatomic periodicity $\left(\mathrm{Fe}_{\mathrm{oct} 2}\right)$. The two remaining Fe layers, consisting of B-site and A-site layers separated by just $0.6 \AA$, can be considered as multilayers $\left(\mathrm{Fe}_{\text {tet } 2} / \mathrm{Fe}_{\text {oct2 }} / \mathrm{Fe}_{\text {tet1 } 1}\right.$ and $\left.\mathrm{Fe}_{\text {oct2 }} / \mathrm{Fe}_{\text {tet } 1}\right)$. Because of this complexity, it is clear that the surface will be sensitive to the preparation conditions.

The experiments on $\mathrm{Fe}_{3} \mathrm{O}_{4}$ described here, were performed in a multichamber ultrahigh vacuum (UHV) system with a base pressure of $2 \times 10^{-10}$ Torr or better. The system was equipped with home-built room-temperature STM, which was fitted with an in-vacuum electromagnet capable of producing an in-plane magnetic field of up to $60 \mathrm{mT}$ without significant thermal drift at the STM head. The system was also equipped with a four-grid reverse view optics for low-energy electron diffraction (LEED) and a cylindrical mirror analyzer for Auger electron spectroscopy (AES). Samples were prepared in a separately pumped chamber, equipped with resistive and electron-beam heaters, an ion source and precision leak valves for introducing high purity gases.

\section{Results and Discussion}

\section{SP-STM on Magnetite (001)}

The $\mathrm{Fe}_{3} \mathrm{O}_{4}(001)$ crystals used in these experiments were grown by the skull melting technique (Harrison and Aragón, 1978). The spinel structure of these crystals was confirmed by powder X-ray diffraction and a lattice constant of $8.40 \pm 0.01 \AA$ was measured. Using resistance vs. temperature curves, a Verwey transition temperature of $108 \mathrm{~K}$ was measured, which indicated that the crystals were substoichiometric (Aragón et al., 1985a, b; Aragón et al., 1986). The crystals were aligned with a precision of $\pm 1^{\circ}$ with respect to the (001) crystallographic plane and mechanically polished to a surface roughness of $0.25 \mu \mathrm{m}$. The in-vacuum preparation consisted of long-cycles of annealing (4-20 h) in UHV at 850-990 K to diffuse contaminants (Ca, $\mathrm{S}, \mathrm{C}$ and $\mathrm{K}$ ) to the surface (Ceballos et al., 2004). These contaminants could be removed by $\mathrm{Ar}^{+}$ion etching (10 min at $1 \mathrm{keV}$ and $10-15 \mu \mathrm{A}$ target current). This was 
followed by annealing for 2-10 h in UHV to re-order the surface and an optional short anneal (1-2 h) in $1 \times 10^{-6}$ Torr $\mathrm{O}_{2}$ to reverse the reducing effects of the UHV anneal.

The resulting surface displayed the well-documented $(\sqrt{ } 2 \times \sqrt{ } 2) \mathrm{R} 45^{\circ} \mathrm{LEED}$ pattern of the clean $\mathrm{Fe}_{3} \mathrm{O}_{4}$ (001) surface, shown in Figure 5 (Voogt et al., 1999; Mijiritskii et al., 2000; Stanka et al., 2000). The surface cleanliness was confirmed by AES. STM images of this surface, also shown in Figure 5, are characterized by rectangular terraces separated by steps aligned along the $<110\rangle$ directions, which are $2.1 \AA$ or integer multiples of this value in height. This value corresponds to the separation between equivalent (001) planes in the bulk $\mathrm{Fe}_{3} \mathrm{O}_{4}$ structure, indicating that the surface terminates at either the A- or B-plane. High-resolution images of the surface show rows of atoms aligned along the $<110>$ directions and separated from one another by a $6 \AA$ spacing. We conclude that the imaged atoms are Fe ions on the basis of photoelectron spectroscopy experiments (Alvarado et al., 1976) and calculations (Zhang and Satpathy, 1991), which demonstrate that the oxygen $2 p$ states lie well below the Fermi level, while the $\mathrm{Fe} 3 d$ states are present near $\mathrm{E}_{\mathrm{F}}$. The rows on terraces separated by $2.1 \AA$ are rotated by $90^{\circ}$ (Figure 5), indicating a two-fold rotational symmetry, which is best described by the B-plane termination of octahedrally coordinated cations.

The atomic structure of these $<110>$ rows is resolved in Figure 6, which shows a series of STM images taken with both MnNi and W tips. The fact that a $12 \AA$ periodicity is clearly observed along the $<110>$ rows with both MnNi and W tips refutes claims in earlier reports that this is a spin-polarized effect that could only be observed when using a magnetic tip (Wiesendanger et al., 1992; Koltun et al., 2001). However, the data obtained with a MnNi tip in Figure 6 do support the conclusion that charge-ordering occurs on the $\mathrm{Fe}_{3} \mathrm{O}_{4}(001)$ surface at room temperature. In the case of the MnNi tip, a periodicity of $12 \AA$ is measured between the corrugation maxima along the $<110>$ rows. An additional $6 \AA$ periodicity can be observed between these maxima and points of lesser corrugation, as indicated in Figure 6 . The full-width at half-maximum (FWHM) measured for the corrugation maxima imaged with the $\mathrm{MnNi}$ tip is of the order of $3 \AA$. The large FWHM combined with the doubling of the periodicity along the $<110>$ rows compared to the interatomic periodicity of B-site cations in the bulk, points towards the formation of cation pairs or dimers on the surface. The STM images were taken with a positive sample bias with respect to the tip, so that the unoccupied electron states in the sample are probed. These maxima are therefore attributed to $\mathrm{Fe}^{3+}-\mathrm{Fe}^{3+}$ cation pairs, while the points of lesser corrugation are attributed to $\mathrm{Fe}^{2+}-\mathrm{Fe}^{2+}$ cation pairs (Mariotto et al., 2002), as shown in Figure 6. We have recently discussed possible driving mechanisms behind the formation of the charge-ordered state on the $\mathrm{Fe}_{3} \mathrm{O}_{4}(001)$ surface (Shvets et al., 2004). Using a W tip, a $12 \AA$ periodicity is also observed along the $<110>$ rows. However, the additional maxima that give rise to the $6 \AA$ periodicity observed with the MnNi tip are not resolved. Instead, the full-width at half-maximum (FWHM) measured for the corrugation maxima imaged with the $\mathrm{W}$ tip is roughly double that measured for the maxima imaged with the $\mathrm{MnNi}$ tip. It should be noted that these results were reproduced in several sessions with different $\mathrm{MnNi}$ and $\mathrm{W}$ tips, so that the issue of tip sharpness is not in question.

To summarise, for both $\mathrm{MnNi}$ and $\mathrm{W}$ tips a $12 \AA$ periodicity in corrugation can be resolved along the $<110\rangle$ cation rows on the B-terminated $\mathrm{Fe}_{3} \mathrm{O}_{4}(001)$ surface. 
This indicates that the measured $12 \AA$ periodicity is simply due to a variation in the LDOS between adjacent $\mathrm{Fe}^{3+}-\mathrm{Fe}^{3+}$ and $\mathrm{Fe}^{2+}-\mathrm{Fe}^{2+}$ cation pairs along the rows rather than a spin-polarized effect. However, with a MnNi tip an additional $6 \AA$ periodicity is observed along the $\left\langle 110>\right.$ rows between the adjacent $\mathrm{Fe}^{3+}-\mathrm{Fe}^{3+}$ and $\mathrm{Fe}^{2+}-\mathrm{Fe}^{2+}$ cation pairs. The enhanced resolution obtained with the MnNi tip may result from the presence of a spin-polarized contribution to the tunnel current.

\section{SP-STM on Magnetite (111)}

The $\mathrm{Fe}_{3} \mathrm{O}_{4}$ (111) crystal was grown by the floating-zone technique (Balbashov and Egorov, 1981). The spinel structure of the crystal was confirmed by highresolution single crystal X-ray diffraction and a lattice constant of $8.3957 \pm 0.0005 \AA$ was measured. From resistivity vs. temperature curves a Verwey transition temperature $\mathrm{T}_{\mathrm{V}}$ of $120 \mathrm{~K}$ was measured. A saturation magnetization of $94 \mathrm{emu} \mathrm{g}^{-1}$ was measured at $297 \mathrm{~K}$ by vibrating sample magnetometry (VSM), which is in good agreement with the value expected for stoichiometric magnetite. The sample was aligned with a precision of $\pm 1^{\circ}$ with respect to the (111) crystallographic plane and mechanically polished to a surface roughness of $0.05 \mu \mathrm{m}$.

The in-vacuum preparation consisted of annealing the sample in UHV at 1000 $\mathrm{K}$ for $100 \mathrm{~h}$ to diffuse contaminants to the surface. This was followed by repeated cycles of $\mathrm{Ar}^{+}$ion etching (5-15 min at 0.5-2.0 keV and 10-15 $\mu \mathrm{A}$ target current) and annealing at $1000 \mathrm{~K}$ in UHV for 3-4 h. Finally, the surface was annealed for $15 \mathrm{~min}$ in $1 \times 10^{-6}$ Torr $\mathrm{O}_{2}$. This procedure produced a contaminant free surface, characterized by a $(1 \times 1)$ hexagonal LEED pattern. STM images of the surface (like the one shown in Figure 7) show terraces separated by monatomic steps of $4.8 \AA$ in height, which is consistent with the separation between equivalent (111) layers in bulk $\mathrm{Fe}_{3} \mathrm{O}_{4}$. An interatomic periodicity of $6 \AA$ was measured on this surface, indicating that it is terminated by tetrahedrally coordinated $\mathrm{Fe}^{3+}$ cations.

The oxygen-terminated (111) surface could be obtained by annealing in $\mathrm{O}_{2}$ for $15 \mathrm{~min}$ and cooling down in the oxygen atmosphere. The resulting surface, also shown in Figure 7, displayed an hexagonal superstructure with a periodicity of $42 \pm 3$ $\AA$, comprising three distinct regions identified as I, II and III. An average interatomic periodicity of $3.1 \pm 0.1 \AA$ was measured in region I, while a periodicity of $2.8 \pm 0.1 \AA$ was measured in regions II and III. Moreover, the height difference between the three regions was found to be strongly bias-dependent. This superstructure represents an oxygen-terminated surface, which reconstructs due to some electron-lattice type instability (Shvets et al., 2003). Oxygen vacancies can typically be observed in this surface layer, and it has been found that above a critical density of these defects $(\geq 20$ $\%$ ), the superstructure does not form (Berdunov et al., 2004a).

SP-STM experiments were performed on this surface, using MnNi tips, by measuring the atomic corrugation of the surface with and without an external magnetic field of $60 \mathrm{mT}$ applied parallel to the sample surface (Berdunov et al., 2004b). We expect the sample has an out-of-plane magnetic moment, along the [111] easy axis, without applying the magnetic field and is in-plane with the magnetic field on, while the magnetization of the tip does not change due to its antiferromagnetic nature. Unfortunately, the magnetostriction effect in magnetic materials like magnetite does not allow one to switch the magnetic field during the scan. The STM images 
were taken on the same sample within the space of a few hours between the nonmagnetic and magnetic experiments. No additional sample treatment between the experiments was performed. The sample was subsequently scanned without the magnetic field to ensure that there was no significant contaminant adsorption during the experiment.

Atomically resolved STM images of the superstructure with and without the external magnetic field are shown in Figure 8. It is clear that the measured atomic corrugation of the superstructure is only affected around oxygen vacancies. Typically, three corrugation maxima appear in a triangular arrangement around each defect. These maxima have a nearest-neighbor separation of $6 \AA$, which corresponds to the positions of $\mathrm{Fe}$ ions in the (111) layer underneath the oxygen termination. There is a corrugation difference of $0.3 \AA$ between these maxima and the surrounding atomic structure.

Density functional theory (DFT) calculations were performed on an oxygenterminated magnetite (111) slab, where the oxygen caps a layer of B-site cations, using the CASTEP simulation with the local spin-density approximation (Berdunov et al., 2004b). The results of these calculations, shown in Figure 9, indicate that the surface oxygen $2 p$ states are sufficiently shifted with respect to $\mathrm{E}_{\mathrm{F}}$, by their strong hybridization with the Fe $3 d$ states, to contribute to the tunnel current at the surface. The effect is already lost in the next oxygen (111) layer, where the $\mathrm{O} 2 p$ states correspond to those found in the bulk. As the images shown in Figure 8 were taken at a sample bias of $-1.0 \mathrm{~V}$, they represent a significant spin polarization at $1 \mathrm{eV}$ below $\mathrm{E}_{\mathrm{F}}$. The spin polarization of the oxygen is much smaller than that of the $\mathrm{Fe}$, which shows majority spin states at $1 \mathrm{eV}$ and $2 \mathrm{eV}$ below $\mathrm{E}_{\mathrm{F}}$. An example of how the surface states change in the presence of the oxygen vacancy is also shown in Figure 9. The calculations were performed for a $2 \times 2$ supercell with and without a surface defect present. Figure 9 shows the majority states distribution above the surface, which displays a maximum density above each oxygen atom in the case of the defect-free surface. In the presence of the oxygen vacancy, we see that new maximas appear and the distribution is no longer regular around the vacancy.

Although, the comparison of the calculations with the experiment is rather qualitative, the main conclusion that can be drawn is that the spin polarization at the magnetite interface is altered locally by the presence of surface defects. One can therefore conclude that the spin-polarized tunneling from the surface represents contributions from both the $\mathrm{Fe}$ and $\mathrm{O}$.

\section{Conclusions}

The atomic structure of the $\mathrm{Fe}_{3} \mathrm{O}_{4}(001)$ and (111) surfaces has been studied by STM, using tips made from antiferromagnetic $\mathrm{MnNi}$. A sharp $(\sqrt{ } 2 \times \sqrt{2}) \mathrm{R} 45^{\circ}$ surface superlattice was observed on the clean $\mathrm{Fe}_{3} \mathrm{O}_{4}(001)$. This is explained in terms of charge ordering at the octahedral sites with the formation of $\mathrm{Fe}^{2+}-\mathrm{Fe}^{2+}$ and $\mathrm{Fe}^{3+}-\mathrm{Fe}^{3+}$ dimers. The difference in corrugation measured on this surface with a $\mathrm{MnNi}$ tip and a $\mathrm{W}$ tip is discussed in terms of a possible spin-polarized tunneling effect. An hexagonal superlattice with $42 \AA$ periodicity is atomically resolved on the oxygenterminated $\mathrm{Fe}_{3} \mathrm{O}_{4}(111)$ surface. In the presence of an external magnetic field, the atomic corrugation of the surface changes in the vicinity of vacancies in the topmost 
oxygen layer. This indicates that defects break the hybridization between the oxygen layer and the underlying Fe layer.

\section{Acknowledgments}

This work was supported by Science Foundation Ireland under contract 00/PI.1/C042 and the EU project ASPRINT (NMP-CT-2003-001601). The authors would like to thank Prof. H. van Kempen for the experiments on $\mathrm{Mn} / \mathrm{Fe}(001)$.

\section{References}

Ahdjoudj J, Martinsky C, Minot C, van Hove MA, Somorjai GA. 1999. Theoretical study of the termination of the $\mathrm{Fe}_{3} \mathrm{O}_{4}(111)$ surface. Surf Sci 443:133-153.

Alvarado SF, Erbudak M, Munz P. 1976. Final-state effects in the $3 d$ photoelectron spectrum of $\mathrm{Fe}_{3} \mathrm{O}_{4}$ and comparison with $\mathrm{Fe}_{\mathrm{x}} \mathrm{O}$. Phys Rev B 14:2740-2745.

Aragón R, Buttrey DJ, Shepherd JP, Honig JM. 1985a. Influence of nonstoichiometry on the Verwey transition. Phys Rev B 31:430-436.

Aragón R, Shepherd JP, Koenitzer JW, Buttrey DJ, Rasmussen RJ, Honig JM. 1985b. Influence of nonstoichiometry on the Verwey transition in $\mathrm{Fe}_{3(1-\delta)} \mathrm{O}_{4}$. $\mathrm{J}$ Appl Phys 57:3221-3222.

Aragón R, Rasmussen RJ, Shepherd JP, Koenitzer JW, Honig JM. 1986. Effect of stoichiometry changes on electrical properties of magnetite. J Magn Magn Mater 5457:1335-1336.

Balbashov AM, Egorov SK. 1981. Apparatus for growth of single crystals of oxide compounds by floating zone melting with radiation heating. J Crystal Growth 52:498504.

Berdunov N, Murphy S, Mariotto G, Shvets IV. 2004a. Room temperature study of a strain-induced electronic superstructure on a magnetite (111) surface. Phys Rev B 70:085404 1-7.

Berdunov N, Murphy S, Mariotto G, Shvets IV. 2004b. Atomically resolved spindependent tunneling on the oxygen-terminated $\mathrm{Fe}_{3} \mathrm{O}_{4}(111)$. Phys Rev Lett 93:057201 $1-4$.

Ceballos SF, Mariotto G, Murphy S, Shvets IV. 2003. Fabrication of magnetic STM probes and their application to studies of the $\mathrm{Fe}_{3} \mathrm{O}_{4}(001)$ surface. Surf Sci 523:131140.

Ceballos SF, Mariotto G, Jordan K, Murphy S, Seoighe C, Shvets IV. 2004. An atomic scale STM study of the $\mathrm{Fe}_{3} \mathrm{O}_{4}(001)$ surface. Surf Sci 548:106-116.

Coey JMD, Shvets IV, Wiesendanger R, Güntherodt H-J. 1993. Charge freezing and surface anisotropy on magnetite (100). J Appl Phys 73:6742-6744. 
Harrison HR, Aragón R. 1978. Skull melter growth of magnetite $\left(\mathrm{Fe}_{3} \mathrm{O}_{4}\right)$. Mat Res Bull 13:1097-1104.

Iida S, Mizushima K, Mizoguchi M, Kose K, Kato K, Yanai K, Goto N, Yumoto S. 1982. Details of the electronic superstructure of $\mathrm{Fe}_{3} \mathrm{O}_{4}$. J Appl Phys 53:2164-2166.

Iida S, Mizoguchi M, Goto N, Motomura Y. 1983. Structure of the anti-phases in the low temperature phase of $\mathrm{Fe}_{3} \mathrm{O}_{4}$. J Magn Magn Mater 31-34:771-772.

Iijima T, Yasuda K. 1988. Submicron-scale tip fabrication for magnetic force microscopy by electrolytic polishing. Jpn J Appl Phys 27:1546-1547.

Jansen R, Brabers VAM, van Kempen H. 1995. One-dimensional reconstruction observed on $\mathrm{Fe}_{3} \mathrm{O}_{4}(110)$ by scanning tunneling microscopy. Surf Sci 328:237-247.

Kasper JS, Kouvel JS. 1959. The antiferromagnetic structure of NiMn. J Phys Chem Solids 11:231-238.

Kita E, Tokuyama Y, Tasaki A, Siratori K. 1983. Possible ionic configurations in the low temperature phase of $\mathrm{Fe}_{3} \mathrm{O}_{4}$. J Magn Magn Mater 31-34:787-788.

Koltun R, Herrmann M, Güntherodt G, Brabers VAM. 2001. Enhanced atomic-scale contrast on $\mathrm{Fe}_{3} \mathrm{O}_{4}$ (100) observed with an Fe STM tip. Appl Phys A 73:49-53.

Kubetzka A, Bode M, Pietzsch O, Wiesendanger R. 2002 Spin-polarized scanning tunneling microscopy with antiferromagnetic probe tips. Phys Rev Lett 88:057201 14.

Kucza W. 2001. Electrostatically driven charge-ordering in magnetite below the Verwey temperature. Solid State Commun 118:401-405.

Mariotto G, Murphy S, Shvets IV. 2002. Charge ordering on the surface of $\mathrm{Fe}_{3} \mathrm{O}_{4}(001)$. Phys Rev B 66:245426 1-8.

Mijiritskii AV, Langelaar MH, Boerma DO. 2000. Surface reconstruction of $\mathrm{Fe}_{3} \mathrm{O}_{4}$ (100). J Magn Magn Mater 211:278-282.

Minakov AA, Shvets IV. 1990. On the possibility of resolving quantization axes of surface spins by means of a scanning tunneling microscope with a magnetic tip. Surf Sci 236:L377-L381.

Mizoguchi M. 1978a. NMR study of the low temperature phase of $\mathrm{Fe}_{3} \mathrm{O}_{4}$. I. Experiments. J Phys Soc Japan 44:1501-1511.

Mizoguchi M. 1978b. NMR study of the low temperature phase of $\mathrm{Fe}_{3} \mathrm{O}_{4}$. II. Electron ordering analysis. J Phys Soc Japan 44:1512-1520.

Molotkov SN. 1992. On the theory of spin-dependent tunneling in the scanning tunneling microscope: antiferromagnetic crystal-ferromagnetic tip. Surf Sci 261:7-16. 
Pál L, Krén E, Kádár G, Szabó P, Tarnóczi T. 1968. Magnetic structures and phase transformations in Mn-based CuAu-I type alloys. J Appl Phys 39:538-544.

Pearson WB, Brun K, Kjekshus A. 1965. Equiatomic transition metal alloys of manganese: III The tetragonal NiMn phase. Acta Chem Scand 19:477-484.

Pierce DT, Davies AD, Stroscio JA, Tulchinsky DA, Unguris J, Celotta RJ. 2000. Non-collinear exchange coupling in $\mathrm{Fe} / \mathrm{Mn} / \mathrm{Fe}(001)$ : insight from scanning tunneling microscopy. J Magn Magn Mater 222:13-27.

Sakuma A. 1998. Electronic structures and magnetism of CuAu-type $\mathrm{MnNi}$ and MnGa. J Magn Magn Mater 187:105-112.

Shvets IV, Wiesendanger R, Bürgler D, Tarrach G, Güntherodt H-J, Coey JMD. 1992. Progress towards spin-polarized scanning tunneling microscopy. J Appl Phys 71:5489-5499.

Shvets IV, Berdunov N, Mariotto G, Murphy S. 2003. Formation of the strain-induced electronic superstructure on the magnetite (111) surface. Europhys Lett 63:867-873.

Shvets IV, Mariotto G, Jordan K, Berdunov N, Kantor R, Murphy S. 2004. Longrange charge order on the $\mathrm{Fe}_{3} \mathrm{O}_{4}$ (001) surface. Phys Rev B 70:155406 1-7.

Slonczewski JC. 1989. Conductance and exchange coupling of two ferromagnets separated by a tunneling barrier. Phys Rev B 39:6995-7002.

Stanka B, Hebenstreit W, Diebold U, Chambers SA. 2000. Surface reconstruction of $\mathrm{Fe}_{3} \mathrm{O}_{4}$ (100). Surf Sci 448:49-63.

Tulchinsky DA, Unguris J, Celotta RJ. 2000. Growth and magnetic oscillatory exchange coupling of $\mathrm{Mn} / \mathrm{Fe}(001)$ and $\mathrm{Fe} / \mathrm{Mn} / \mathrm{Fe}(001)$. J Magn Magn Mater 212:91100.

Verwey EJ, Haayman PW, Romeijn FC. 1947 Physical properties and cation arrangement of oxides with spinel structures: II Electronic conductivity. J Chem Phys 15:181-187.

Voogt FC, Fujii T, Smulders PJM, Niesen L, James MA, Hibma T. 1999. $\mathrm{NO}_{2-}$ assisted molecular-beam epitaxy of $\mathrm{Fe}_{3} \mathrm{O}_{4}, \mathrm{Fe}_{3-\delta} \mathrm{O}_{4}$, and $\gamma-\mathrm{Fe}_{2} \mathrm{O}_{3}$ thin films on MgO(100). Phys Rev B 60:11193-11206.

Walz F. 2002. The Verwey transition - a topical review. J Phys: Cond Matter 14:R285-R340

Wiesendanger R, Shvets IV, Bürgler D, Tarrach G, Güntherodt HJ, Coey JMD, Gräser S. 1992. Topographic and magnetic-sensitive scanning tunneling microscope study of magnetite. Science 255:583-586.

Wyckoff RWG. 1965. Crystal Structures vol. 3. London: Interscience. p 79. 
Yamada TK, Bischoff MMJ, Heijnen GMM, Mizoguchi T, van Kempen H. 2003. Observation of spin-polarized surface states on ultrathin bet Mn(001) films by spinpolarized scanning tunneling spectroscopy. Phys Rev Lett 90:056803 1-4.

Zhang Z, Satpathy S. 1991. Electron states, magnetism, and the Verwey transition in magnetite. Phys Rev B 44:13319-13331.
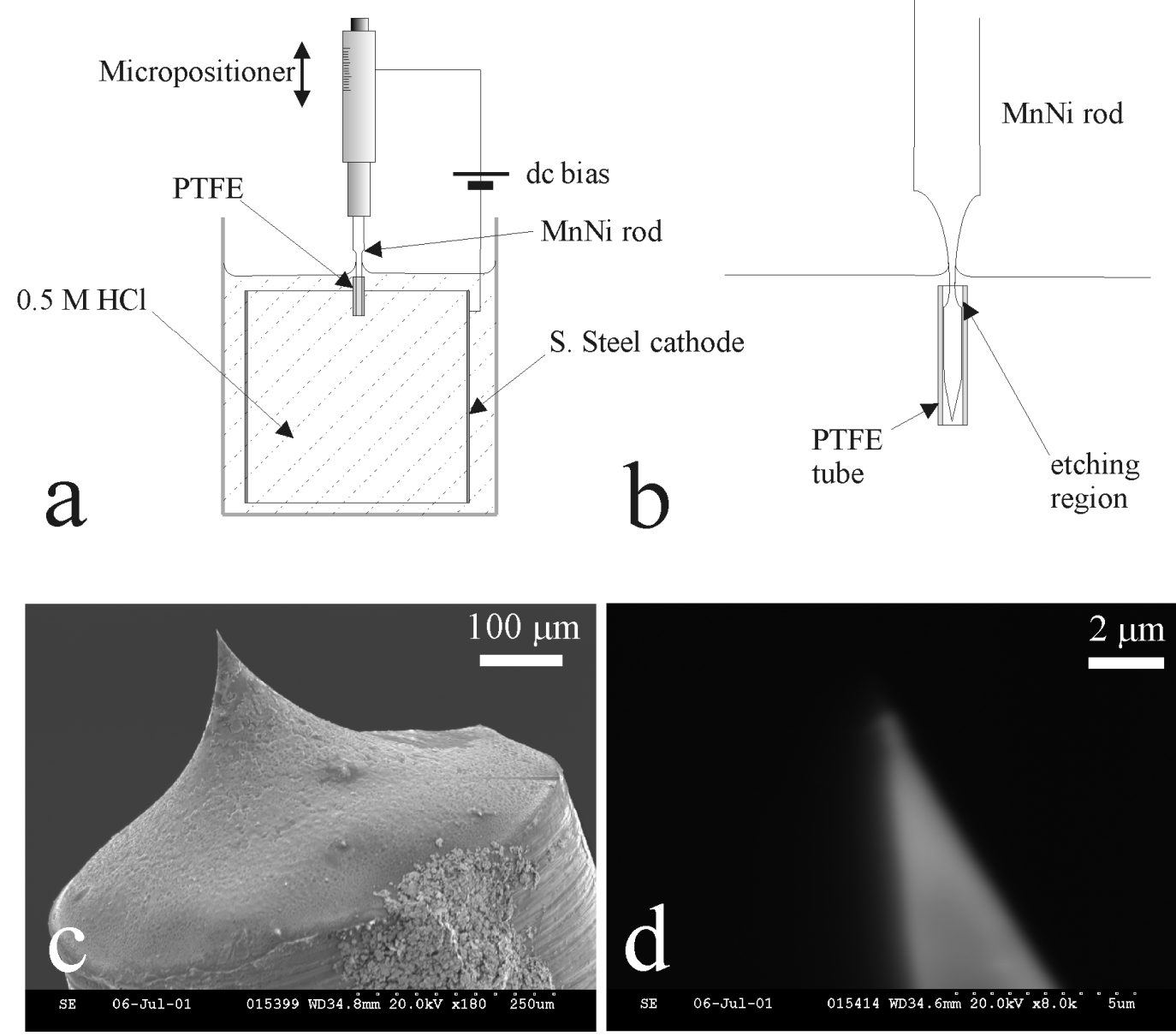

Fig. 1. Technique for restricting the active etching region when etching MnNi tips. (a) The lower portion of the MnNi rod is sheathed in a PTFE tube and immersed in a bath of 0.5 molar HCl. A stainless steel foil is used as the counter-electrode. (b) The tip is formed within the upper end of the PTFE tube. (c, d) SEM micrographs of a MnNi tip etched using the PTFE tubing method. The scale is indicated in each image. The residue on the tip shown in image (c) is a by-product of the electrochemical etch and is later removed in vacuum by ion etching. 


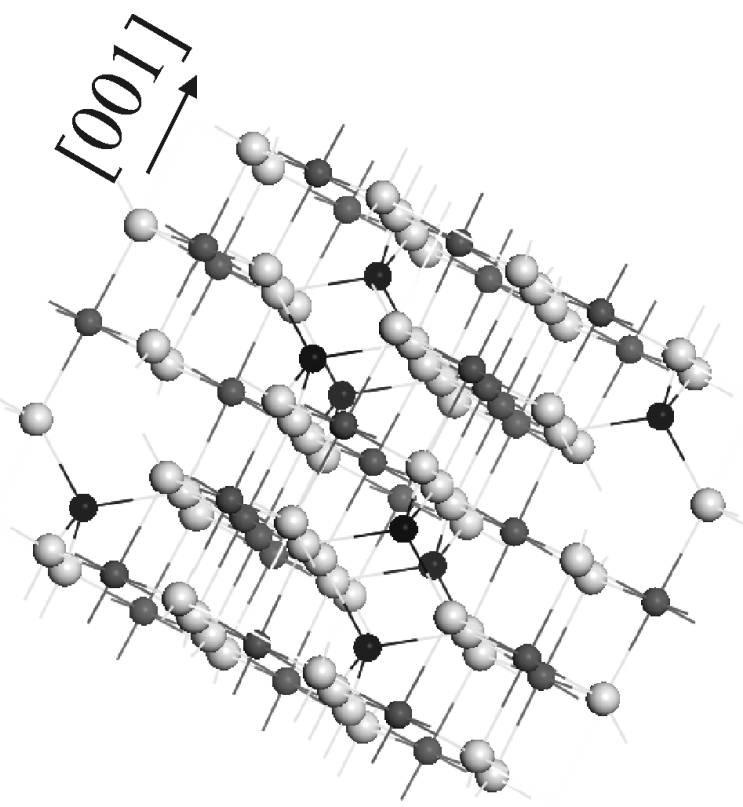

Fig. 2. Model of the magnetite inverse spinel crystal structure. Light gray circles represent oxygen anions, while dark gray circles correspond to B-site cations and black circles correspond to A-site cations. The lattice parameter of the unit cell is $8.3963 \AA$ (Wyckoff, 1965).

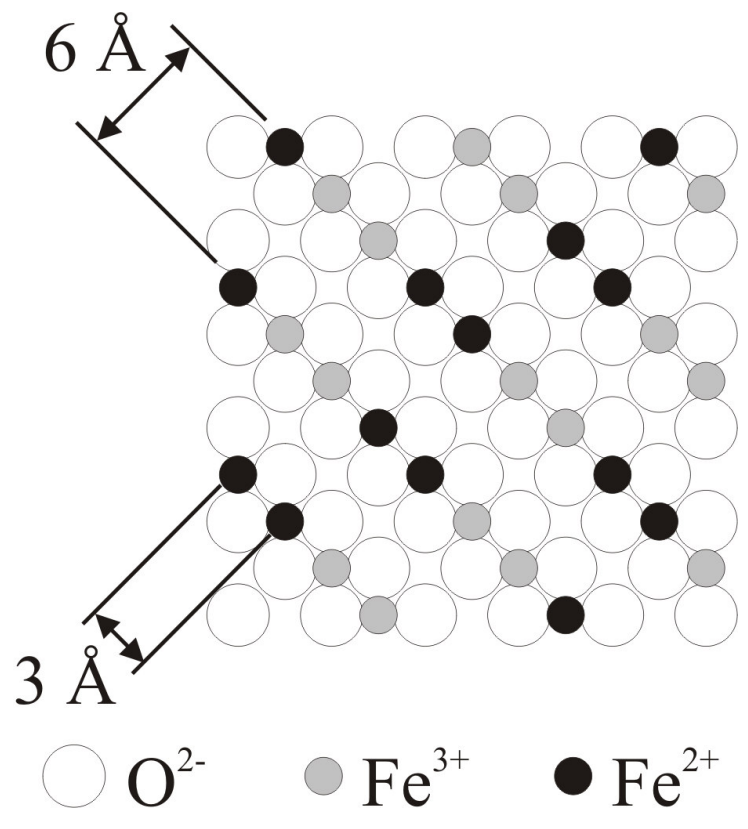

Fig. 3. Proposed model for the charge ordered B-terminated $\mathrm{Fe}_{3} \mathrm{O}_{4}(001)$ surface (Wiesendanger et al., 1992). The orientation of the $\mathrm{Fe}<110>$ rows rotates by $90^{\circ}$ between successive octahedral planes, which are separated by an interlayer distance of $2.1 \AA$. 


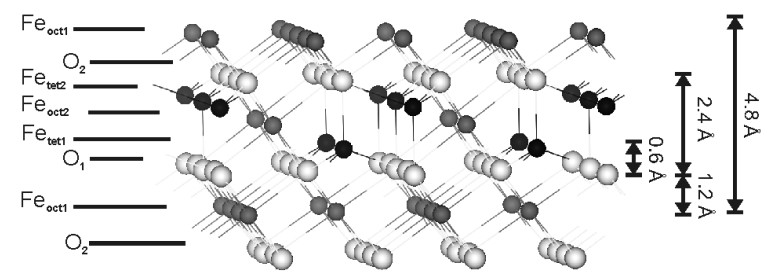

Fig. 4. Tilted view of the $\mathrm{Fe}_{3} \mathrm{O}_{4}$ crystal structure, showing the six different (111) layers of the unit cell. The large gray circles represent oxygen anions, the small gray circles are B-site cations and the small dark circles are A-site cations. The interlayer separations between various layers are also given.
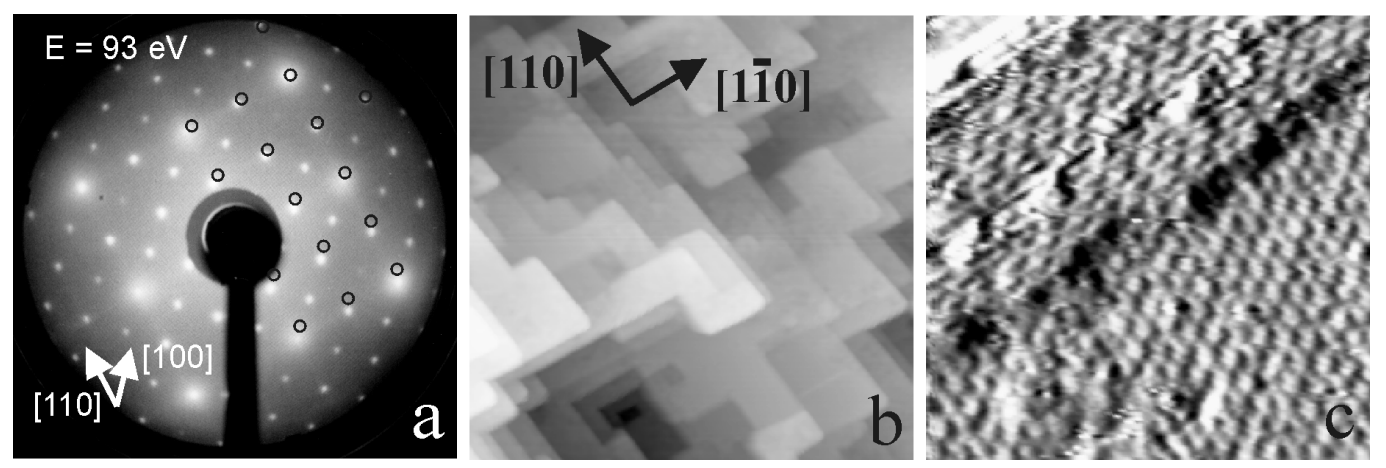

Fig. 5. (a) LEED pattern of the clean magnetite (001) surface, showing the characteristic $(\sqrt{2} \times \sqrt{2}) \mathrm{R} 45^{\circ}$ superlattice. The integral order spots are highlighted on one side of the screen. (b) STM images of the clean $\mathrm{Fe}_{3} \mathrm{O}_{4}(001)$ surface. $100 \mathrm{~nm} \times$ $100 \mathrm{~nm}$ image showing square terraces separated by $2.1 \AA$ steps, which are aligned along the $<110>$ directions. (c) $15 \mathrm{~nm} \times 15 \mathrm{~nm}$ differentiated image showing $<110>$ oriented atomic rows which are rotated by $90^{\circ}$ on successive terraces separated by a $2.1 \AA$ step, indicating that the termination is most likely comprised of octahedrally coordinated Fe cations.
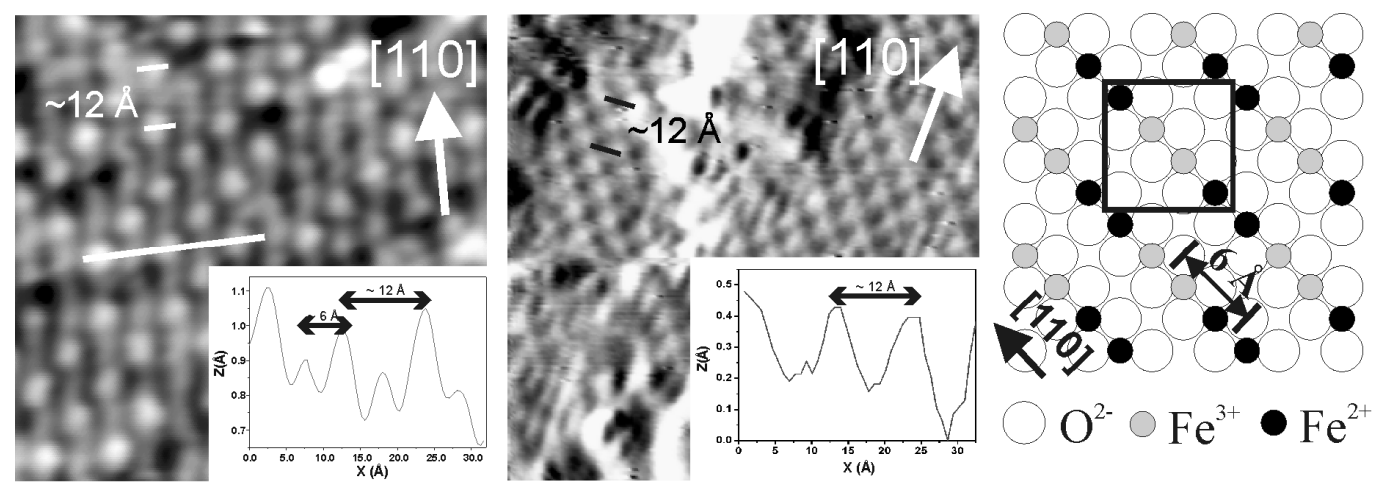

Fig. 6. Atomic structure of the $<110>$ rows imaged ( $\mathrm{V}=1.0 \mathrm{~V}$ and $\mathrm{I}=0.1 \mathrm{nA}$ ) with $\mathrm{MnNi}$ and $\mathrm{W}$ tips respectively. (left) $7 \mathrm{~nm} \times 7 \mathrm{~nm}$ image taken with a MnNi tip, the [1-10] line profile highlights the additional $6 \AA$ periodicity. (middle) $13 \mathrm{~nm} \times 13 \mathrm{~nm}$ differentiated image of the $<110>$ rows taken with a W tip, showing the $12 \AA$ periodicity. (right) Model proposed for the charge-ordered $(\sqrt{2} \times \sqrt{2}) \mathrm{R}_{4} 5^{\circ} \mathrm{Fe}_{3} \mathrm{O}_{4}(001)$ surface (Mariotto et al., 2002). 

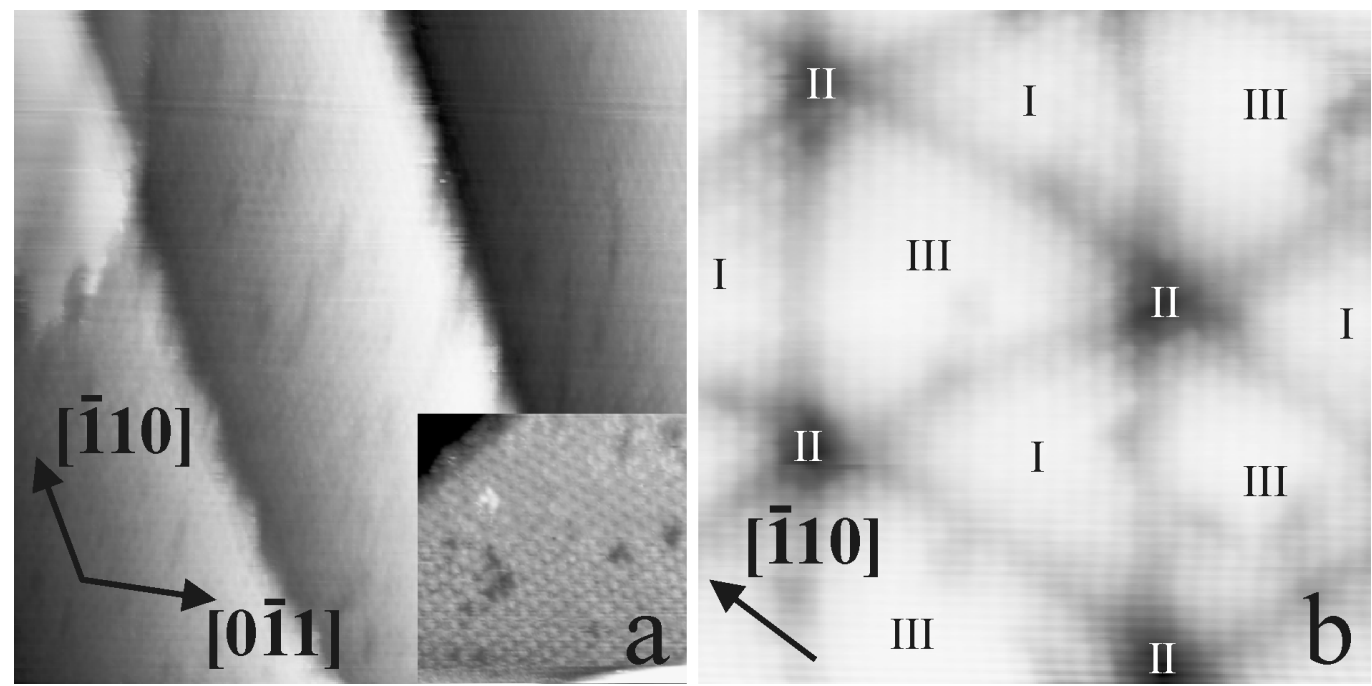

Fig. 7. (a) $50 \mathrm{~nm} \times 50 \mathrm{~nm}$ STM image of the clean magnetite (111) surface, showing terraces separated by $4.8 \AA$ high steps. The insert shows a $11 \mathrm{~nm} \times 11 \mathrm{~nm}$ atomically resolved image. The interatomic separation is $6 \AA$, which corresponds to a termination of tetrahedrally coordinated $\mathrm{Fe}^{3+}$ cations. (b) $8 \mathrm{~nm} \times 8 \mathrm{~nm}$ STM image of the patterned magnetite (111) surface. The hexagonal superstructure has a periodicity of $42 \pm 3 \AA$, with three distinct regions of atomic corrugation identified as I, II and III. The average interatomic separation measured in region $\mathrm{I}$ is $3.1 \pm 0.1 \AA$, while it is 2.8 $\pm 0.1 \AA$ in regions II and III.
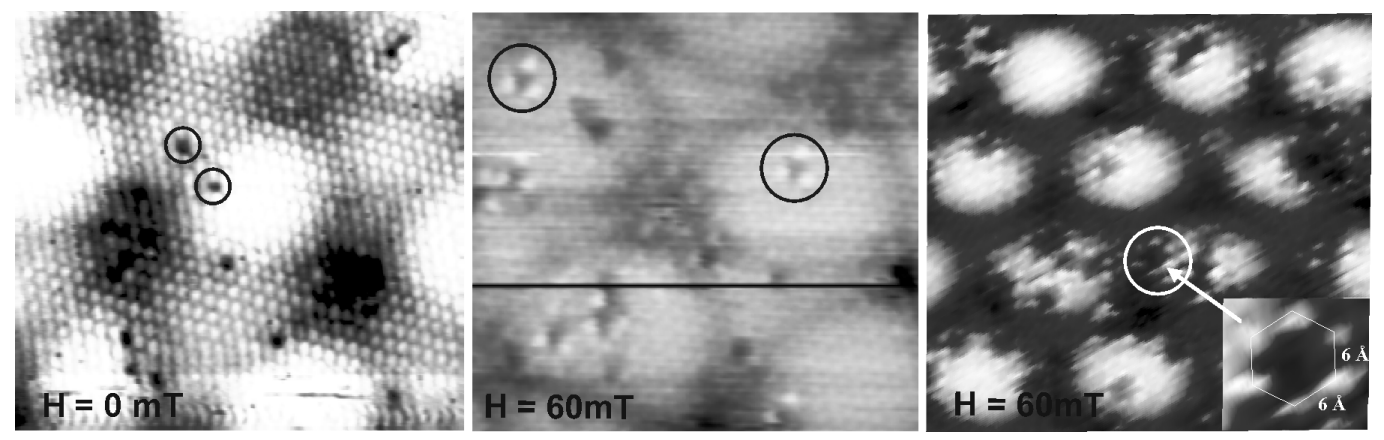

Fig. 8. Effect of an applied magnetic field on oxygen vacancies imaged on the $\mathrm{Fe}_{3} \mathrm{O}_{4}(111)$ surface by STM using a MnNi tip. (left) $10.5 \mathrm{~nm} \times 8.5 \mathrm{~nm}$ image of single vacancies in zero applied field, (middle) $7 \mathrm{~nm} \times 8 \mathrm{~nm}$ image taken with an applied field of $60 \mathrm{mT}$, showing three bright points surrounding each single vacancy and (right) $12 \mathrm{~nm} \times 17.5 \mathrm{~nm}$ image taken in the same field with an example of an hexagonal multiple defect. All three images were taken using the same tunnel parameters of $\mathrm{V}=-1.0 \mathrm{~V}$ and $\mathrm{I}=0.1 \mathrm{nA}$. 

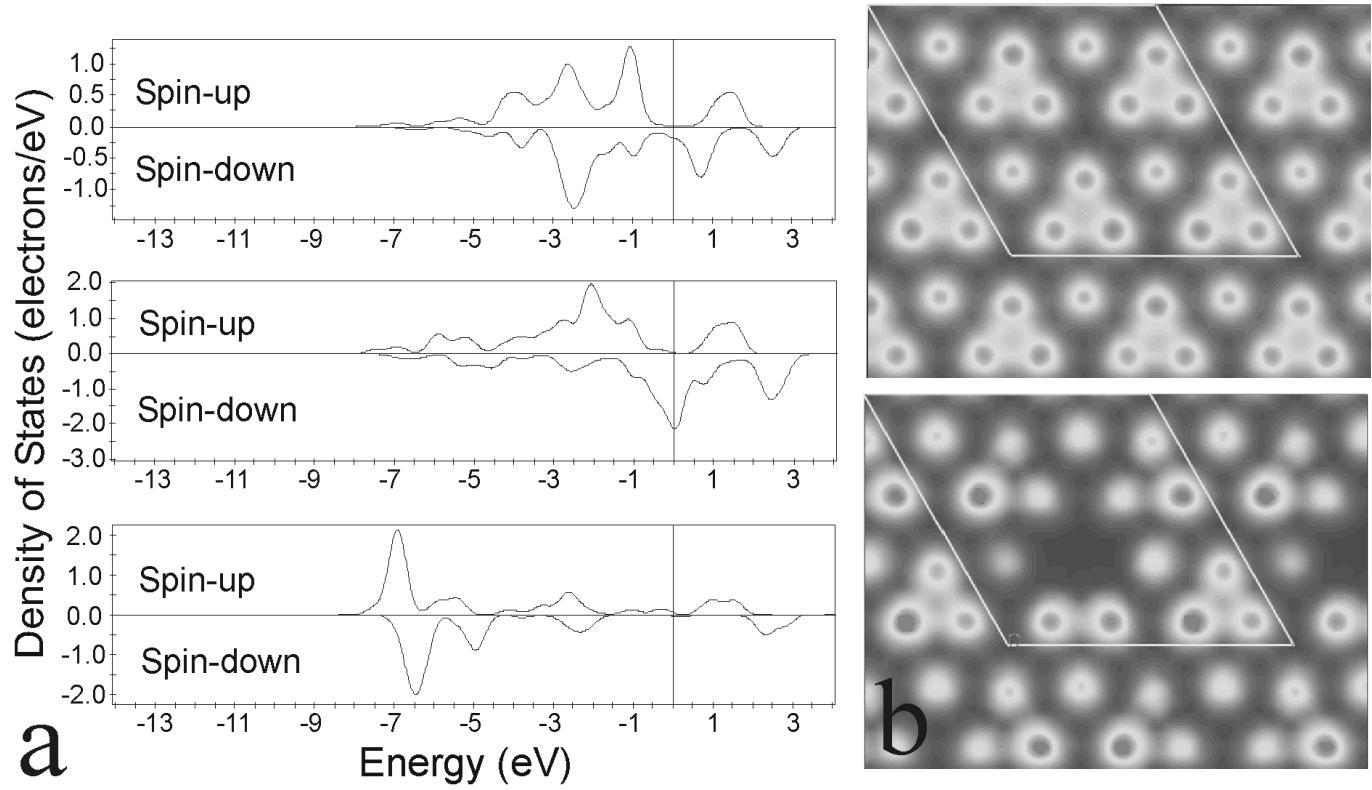

Fig. 9. Results of DFT calculations for the $\mathrm{Fe}_{3} \mathrm{O}_{4}(111)$ surface. (a) Layer-projected partial density of states for (top) $\mathrm{O} 2 p$ states in a close-packed oxygen topmost layer, (middle) Fe $3 d$ states in an octahedrally coordinated Fe layer and (bottom) $\mathrm{O} 2 p$ states in the third layer. (b) The spin-up electron density above an ideal oxygen-terminated surface (top) and in the presence of an oxygen vacancy (bottom). The displayed energy range $\left[\mathrm{E}_{\mathrm{F}}-1, \mathrm{E}_{\mathrm{F}}\right]$ is compatible with that used for the STM experiments. The $(2 \times 2)$ supercell used for the calculation is marked. 